\title{
CÁC ÚNG DỤNG RộNG RÃI CỦA LÝ THUYẾT MOLODENXKII M.X. TRONG TRẮC ĐỊA VẬT LÝ TRÊN THẾ GIỚI
}

\author{
HÀ MINH HÒA \\ Viện Khoa học Đo đạc và Bản đồ
}

\section{Tóm tắt:}

Vấn đề mấu chốt để xây dựng hệ quy chiếu không gian quốc gia là xây dụng mô hình quasigeoid độ chính xác cao. Tuy nhiên hiện nay giữa các nhà trắc địa ở Việt Nam vẫn nhầm lẫn các khái niệm về quasigeoid và geoid. Trong bài báo này, trên co sở nghiên cúu các đặc trung co bản của lý thuyết Molodenxkii M.X., chúng ta sẽ làm rõ khái niệm về độ cao chuẩn, dị thương độ cao và các xu huớng sư dụng rộng rãi lý thuyết này trên thế giới.

\section{1. Đặt vấn đề}

Vào năm 1849, Stokes G. đã đề xuất bài toán biên hỗn hợp của Trắc địa vật lý, theo đó mặt biên là mặt geoid đồng thời là mặt khởi tính của hệ độ cao chính, ở đây độ cao chính $H_{P}^{g}$ của điểm p trên mặt vật lý Trái đất là khoảng cách theo đường sức của lực trọng trường từ điểm $\mathrm{P}$ đến điểm $\mathrm{Q}$ trên mặt geoid, còn độ cao geoid $N_{P}$ của điểm $\mathrm{p}$ là khoảng cách từ điểm $\mathrm{Q}$ đến mặt ellipsoid quy chiếu. Nghiệm của bài toán biên là các giá trị thế nhiễu được xác định trên mặt biên nhận được từ việc giải phương trình tích phân biên của Stokes G. (Stokes, G.G., 1849). Do thế nhiễu là hàm điều hòa trên mặt biên và trong không gian ngoài mặt biên, tức ở đó hàm điều hòa là hàm liên tục, có các đạo hàm các bậc và hàm điều hòa bằng 0 tại điểm vô cực trong không gian ngoài mặt biên, nên luôn đặt ra yêu cầu để trên mặt biển không tồn tại bất kỳ khối lượng vật chất nào. Khi giải quyết bài toán biên hỗn hợp của Trắc địa vật lý theo cách tiếp cận của Stokes G., do mặt geoid nằm sâu trong lớp vỏ Trái đất, nên nẩy sinh hai vấn đề của Stokes G.:

Vấn đề 1. Phải loại bỏ toàn bộ khối lượng vật chất của các lục địa, các quần đảo và các đảo nằm trên mặt geoid và đưa các khối lượng vật chất vào dưới mặt geoid.

Vấn đề này được giải quyết nhờ phương pháp cô đặc thứ hai của Helmert. Trong trường hợp này, các giá trị dị thường trọng lực được quy chiếu lên mặt geoid là các dị thường Helmert.

Vấn đề 2. Phải biết quy luận phân bố mật độ vật chất trong lớp vỏ Trái đất để quy chiếu các giá trị gia tốc lực trọng trường đo được trên mặt vật lý Trái đất xuống mặt geoid.

Vấn đề thứ hai là vấn đề nan giải cho đến ngày hôm nay. Đây là nguyên nhân cơ bản hạn chế sự phổ biến của phương pháp Stokes G. trong thực tế nghiên cứu trọng trường Trái đất. Để khắc phục các vấn đề của Stokes G., bắt đầu từ các công trình (Molodenxkii, M.X., 1945; Molodenxkii, M.X., 1948), khi lưu ý các đặc điểm sau: Ellipsoid Trái đất cùng quay với Trái đất, có khối lượng bằng khối lượng trung bình $\mathrm{M}$ của Trái đất, có tốc độ quay bằng tốc độ quay trung bình 0 của Trái đất, thế trọng trường chuẩn $U$ của điểm trên mặt vật lý Trái đất chênh với thế trọng trường thực $W$ của điểm đó một đại lượng không lớn được gọi là thế nhiễu $T=W-U$ Molodenxkii M.X. đã đề xuất đưa bài toán biên hỗn hợp của Trắc địa vật lý vào trọng trường chuẩn của ellipsoid với mục đích tránh việc phải biết quy luật phân bố mật độ vật chất của lớp vỏ Trái đất (Heiskanen W.A. and Moritz H., 1967; Simberov, B.P., 1975; Jekeli C., 2000; Matt, A., 2010; Hirt C., Featherstone W.E. Marti U., 2010; Ogorodova L.V., 2010). Như vậy vấn đề thứ hai của Stokes G. được giải quyết.

Ngày nhận bài: 04/9/2018, ngày chuyển phản biện: 07/9/2018, ngày chấp nhận phản biện: 10/9/2018, ngày chấp nhận đăng: 14/9/2018 
Để giải quyết vấn đề thứ nhất của Stokes G., Molodenxkii M.X. xác định mặt biên của bài toán biên sao cho nó gần với mặt vật lý Trái đất. Đối với điểm $\mathrm{P}$ trên mặt vật lý Trái đất, chúng ta ký hiệu $H_{P}^{\gamma}$ là độ cao chuẩn và $\zeta_{P}$ là dị thường độ cao của điểm theo lý thuyết của Molodenxkii M.X., thêm vào đó các đại lượng này được xác định trong trọng trường chuẩn của ellipsoid quy chiếu (Simberov, B.P., 1975; Ogorodova L.V., 2010). Do các độ cao của các điểm trên mặt vật lý Trái đất lớn hơn rất nhiều so với dị thường độ cao, ví dụ độ cao chuẩn của đỉnh Everest thuộc dãy Hymalaya bằng 8848 $\mathrm{m}$, trong khi đó theo các dữ liệu hiện đại của mô hình trọng trường Trái đất EGM2008 (NGA.STND.0036_1.0.0_WGS84), các giá trị dị thường độ cao toàn cầu tương ứng với ellipsoid WGS84 đạt giá trị cực tiểu - 106,91 m và giá trị cực đại $+85,82 \mathrm{~m}$, nên để đạt được mục đích đề ra, trên đường vuông góc từ điểm $\mathrm{P}$ đến mặt ellipsoid, từ điểm $\mathrm{Q}_{0}$ trên mặt ellipsoid dựng đoạn $\mathrm{Q}_{0} \mathrm{~N}$ bằng độ cao chuẩn $H_{P}^{\gamma}$. Khi đó đoạn NP bằng dị thường độ cao $\zeta_{P}$ (xem Hình 1$)$. Đối với tập hợn các điểm $\mathrm{P}$ trên mặt vật lý Trái đất, quỹ tích các điểm $\mathrm{N}$ tạo thành một mặt được gọi là mặt telluroid. Do mặt telluroid khá gần với mặt vật lý Trái đất, nên Molodenxkii M.X. đã sử dụng nó làm mặt biên để giải quyết bài toán biên hỗn hợp của Trắc địa vật lý.

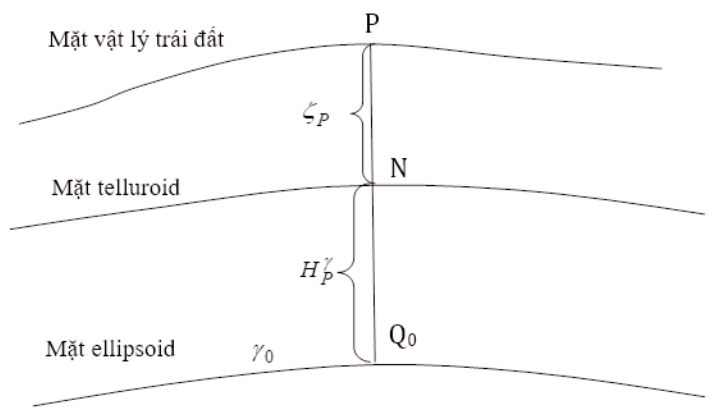

Hình 1: Mặt telluroid

Chúng ta cũng cần thiết phải nhấn mạnh rằng trong lý thuyết của Molodenxkii M.X. tồn tại hai mặt: Mặt telluroid để giải quyết quyết bài toán biên hỗn hợp của Trắc địa vật lý và mặt quasigeoid để xây dựng lý thuyết hệ độ cao chuẩn cho phù hợp với lý thuyết độ cao của Listing J.B. (Listing, J.B., 1873), theo đó mặt khởi tính hệ độ cao phải là mặt geoid. Việc nghiên cứu mặt quasigeoid sẽ được xem xét trong bài báo tiếp theo. Tuy nhiên, do độ cao chuẩn $H_{P}^{\gamma}$ và dị thường độ cao $\zeta_{z}$ được xác định trong trọng trường chuẩn của ellipsoid, nên từ Hình 1 chúng ta thấy rằng chúng được xác định theo các công thức sau:

$$
H_{P}^{\gamma}=\frac{U_{0}-U_{N}}{\bar{\gamma}_{P}},
$$

$$
\zeta_{P}=\frac{U_{N}-U_{P}}{\bar{\gamma}_{P}},
$$

ở đây $U_{0}$ - thế trọng trường chuẩn trên mặt ellipsoid; giá trị trung bình của gia tốc lực trọng trường chuẩn $\bar{y}_{s}$ tại điểm $\mathrm{P}$ được xác định theo công thức:

$$
\bar{\gamma}_{P}=\gamma_{0}-0,1543 \cdot(1+0,0007 \cdot \cos 2 B) \cdot H_{M}^{\gamma}+0,036 \cdot 10^{-6} \cdot\left(H_{M}^{\gamma}\right)^{2},
$$

$\gamma_{0}$ - gia tốc lực trọng trường chuẩn trên mặt ellipsoid.

Các vấn đề được đặt ra cần được làm rõ khi nghiên cứu lý thuyết của Molodenxkii M.X. là khái niệm về độ cao chuẩn, lý giải việc sử dụng mặt vật lý Trái đất làm mặt biên trong thực tế giải bài toán biên hỗn hợp và các xu hướng ứng dụng lý thuyết này. Việc giải quyết các vấn đề nêu trên là 
các nội dung của bài báo khoa học này.

\section{Giải quyết vấn đề}

\subsection{Tổng quan lý thuyết của Molodenxkii M.X.}

Gia tốc lực trọng trường thực $g_{P}$ tại điểm $\mathrm{P}$ trên mặt vật lý Trái đất quan hệ với gia tốc lực trọng trường thực $g_{N}$ tại điểm $\mathrm{N}$ trên mặt telluroid bởi biểu thức:

$$
g_{P}=g_{N}+\frac{\partial g}{\partial \rho} \cdot \zeta_{P},
$$

còn gia tốc lực trọng trường chuẩn $\mho_{\Im}$ tại điểm $\mathrm{P}$ trên mặt vật lý Trái đất quan hệ với gia tốc lực trọng trường chuẩn $\gamma_{y}$ tại điểm $\mathrm{N}$ trên mặt telluroid bởi biểu thức:

$$
\gamma_{p}=\gamma_{N}+\frac{\partial \gamma}{\partial \rho} \cdot \zeta_{P}
$$

ở đây $\frac{\partial g}{\partial \rho}$ - gradient gia tốc lực trọng trường thực theo bán kính - vector $p$ của lớp vỏ Trái đất giữa điểm $\mathrm{P}$ và điểm $\mathrm{N}$ trong trọng trường thực của Trái đất, $\frac{\partial \gamma}{\partial \rho}=-0,3086 \mathrm{mGal} / \mathrm{m}-$ gradient của gia tốc lực trọng trường chuẩn theo bán kính - vector $p$ trong trọng trường chuẩn của ellipsoid.

Chúng ta biến đổi công thức (3) như sau:

$$
g_{P}=g_{N}+\frac{\partial g}{\partial \rho} \cdot \zeta=g_{N}+\frac{\partial \gamma}{\partial \rho} \cdot \zeta+d=\hat{g}_{P}+d,
$$

ở đây gia tốc lực trọng trường

$$
\hat{g}_{p}=g_{N}+\frac{\partial \gamma}{\partial \rho} \cdot \zeta_{P}
$$

độ lệch

$$
d=\left(\frac{\partial g}{\partial \rho}-\frac{\partial \gamma}{\partial \rho}\right) \zeta
$$

Chúng ta giả thiết rằng do dị thường độ cao 5 đủ nhỏ sao cho giữa điểm $\mathrm{P}$ và điểm $\mathrm{N}$ là "không khí tự do", tức

$$
\frac{\partial g}{\partial \rho} \approx \frac{\partial \gamma}{\partial \rho}
$$

Khi đó độ lệch $\mathrm{d}(7)$ đủ nhỏ và từ (5) suy ra $g_{p} \approx \hat{g}_{p}$. Bây giờ đối với điểm $\mathrm{N}$ trên mặt telluroid, khi lấy đạo hàm thế nhiễu $T_{N}=W_{N}-U_{N}$ theo bán kính vectơ - địa tâm $p$, chúng ta nhận được gradient của thế nhiễu tại điểm $\mathrm{N}$ như sau:

$$
\left.\frac{\partial T_{N}}{\partial \rho}\right|_{N}=\left.\frac{\partial W_{N}}{\partial \rho}\right|_{N}-\left.\frac{\partial U_{N}}{\partial \rho}\right|_{N}=-g_{N}+\gamma_{N} .
$$

Từ (6) và (9) chúng ta nhận được phương trình tích phân của bài toán biên hỗn hợp trên mặt telluroid theo cách tiếp cận của Molodenxkii M.X. ở dạng sau:

$$
\left.\frac{\partial T_{N}}{\partial \rho}\right|_{N}=-\hat{g}_{P}+\gamma_{N}+\frac{\partial \gamma}{\partial \rho} \cdot \zeta_{P}=-g_{P}+\gamma_{N}+\frac{\partial \gamma}{\partial \rho} \cdot \zeta_{P}
$$

hay

$$
\left.\frac{\partial T_{N}}{\partial \rho}\right|_{N}=-\Delta g_{k k t d}+\frac{\partial \gamma}{\partial \rho} \cdot \zeta_{P},
$$



M.X..

ở đây $\Delta g_{k k d d}=g_{P}-\gamma_{N}$ được gọi là dị thường không khí tự do theo cách tiếp cận của Molodenxkii

Chúng ta ký hiệu $\overline{W_{0}}$ là thế trọng trường thực của mặt geoid toàn cầu, $W_{P}$ là thế trọng trường thực tại điểm $\mathrm{P}$ trên mặt vật lý Trái đất. Bây giờ chúng ta nghiên cứu phương trình tích phân biên theo cách tiếp cận của Molodenxkii M.X. trong hai trường hợp.

Trường hợp 1: Ellipsoid được biểu diễn trên Hình 1 là ellipsoid trái đất trung bình (mean Earth ellipsoid, normal Earth) là mô hình toán lý tốt nhất của geoid toàn cầu. Trong trường hợp này, thế trọng trường chuẩn $\bar{F}_{0}$ trên mặt ellipsoid trái đất trung bình thỏa mãn tính chất (Pellinen, L.P., 1978; Hofmann-Wellenhof B. and Moritz H., 2005):

$$
\bar{U}_{0}=\bar{W}_{0} .
$$

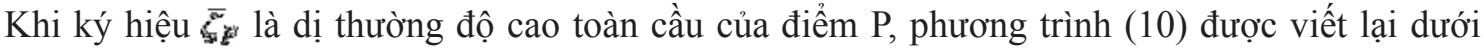
dạng:

$$
\left.\frac{\partial T_{N}}{\partial \rho}\right|_{N}=-\Delta g_{k k t d}+\frac{\partial \gamma}{\partial \rho} \cdot \bar{\zeta}_{P},
$$

Trong trường hợp này, đoạn $\mathrm{PQ}_{0}$ là độ cao trắc địa toàn cầu được xác định theo công thức:

$$
\bar{H}_{P}=\frac{\bar{U}_{0}-U_{P}}{\bar{\gamma}_{P}} \text {. }
$$

Để thiết lập hệ độ cao chuẩn với mặt geoid toàn cầu, Molodenxkii M.X. đã thiết lập điều kiện (Simberov, B.P., 1975):

$$
\bar{U}_{0}-U_{N}=\bar{W}_{0}-W_{P} \text {. }
$$

Khi đó từ công thức (1) chúng ta biểu diễn độ cao chuẩn toàn cầu $\bar{H}_{P}^{\gamma}$ dưới dạng:

$$
\bar{H}_{P}^{\gamma}=\frac{\bar{U}_{0}-U_{N}}{\bar{\gamma}_{P}}=\frac{\bar{W}_{0}-W_{P}}{\bar{\gamma}_{P}} .
$$

Lưu ý quan hệ $\bar{\zeta}_{P}=\bar{H}_{P}-\bar{H}_{P}^{\gamma}$, từ các công thức (13), (15) chúng ta biểu diễn dị thường độ cao toàn cầu dưới dạng công thức Bruns:

$$
\bar{\zeta}_{P}=\bar{H}_{P}-\bar{H}_{P}^{\gamma}=\frac{\bar{U}_{0}-U_{P}}{\bar{\gamma}_{P}}-\frac{\bar{W}_{0}-W_{P}}{\bar{\gamma}_{P}}=\frac{T_{P}}{\bar{\gamma}_{P}}+\frac{\bar{U}_{0}-\bar{W}_{0}}{\bar{\gamma}_{P}},
$$

ở đây $T_{P}=W_{P}-U_{P}$ - thế nhiễu tại điểm $\mathrm{P}$.

Khi rút $U_{N}$ ra từ (14) và thay vào (2), chúng ta cũng sẽ nhận được công thức (16). Khi thay (16) vào (12), chúng ta nhận được phương trình tích phân biên theo cách tiếp cận của Molodenxkii M.X. ở dạng sau:

$$
\left.\frac{\partial T_{N}}{\partial \rho}\right|_{N}-\frac{\partial \gamma}{\partial \rho} \cdot \frac{T_{P}}{\bar{\gamma}_{P}}=-\Delta g_{k k t d}+\frac{\partial \gamma}{\partial \rho} \cdot \frac{\left(\bar{U}_{0}-\bar{W}_{0}\right)}{\bar{\gamma}_{P}} .
$$

Mặt khác, khi lấy đạo hàm thế nhiễu $T_{P}=W_{P}-U_{P}$ tại điểm $\mathrm{P}$ theo bán kính vectơ - địa tâm $p$ chúng ta nhận được gradient của thế nhiễu tại điểm P như sau:

$$
\left.\frac{\partial T_{P}}{\partial \rho}\right|_{P}=\left.\frac{\partial W_{P}}{\partial \rho}\right|_{P}-\left.\frac{\partial U_{P}}{\partial \rho}\right|_{P}=-g_{P}+\gamma_{P}
$$

Thay $q^{2}$ từ (4) vào phương trình trên, chúng ta có:

$$
\left.\frac{\partial T_{P}}{\partial \rho}\right|_{P}=-\Delta g_{k k t d}+\frac{\partial \gamma}{\partial \rho} \cdot \bar{\zeta}_{P} .
$$


Khi so sánh các công thức (12), (18) chúng ta nhận được đẳng thức:

$$
\left.\frac{\partial T_{P}}{\partial \rho}\right|_{P}=\left.\frac{\partial T_{N}}{\partial \rho}\right|_{N} .
$$

Lưu ý (11), (19), trong trường hợp đang xem xét, phương trình tích phân biên (17) theo cách tiếp cận của Molodenxkii M.X. có dạng sau:

$$
\left.\frac{\partial T_{P}}{\partial \rho}\right|_{P}-\frac{\partial \gamma}{\partial \rho} \cdot \frac{T_{P}}{\bar{\gamma}_{P}}=-\Delta g_{k k t d} .
$$

Từ (20), chúng ta thấy rằng mặc dù bài toán biên hỗn hợp theo cách tiếp cận của Molodenxkii M.X. được xây dựng trên mặt telluroid, nhưng dạng cuối cùng của nó lại được xác định trên mặt vật lý Trái đất. Khi lưu ý điều kiện (11) đối với ellipsoid Trái đất trung bình, từ (14) suy ra $U_{N}=W_{P}$. Dựa trên đẳng thức này, trong rất nhiều tài liệu về Trắc địa vật lý, ví dụ Jekeli C., 2000; HofmannWellenhof B. and Moritz H., 2005; Ogorodova L.V., 2010, đã định nghĩa độ cao chuẩn tại điểm $\mathrm{P}$ trên mặt vật lý được xác định trong trọng trường chuẩn của ellipsoid sao cho thế trọng trường thực $W_{P}$ tại điểm này bằng thế trọng trường chuẩn $U_{N}$ tại điểm $\mathrm{N}$ tương ứng với điểm $\mathrm{P}$ và nằm trên mặt telluroid.

Tuy nhiên chúng ta cần thiết phải nhấn mạnh rằng định nghĩa nêu trên chỉ đúng trong trường hợp sử dụng ellipsoid Trái đất trung bình. Vì lý do này, chúng ta sẽ nghiên cứu trường hợp thứ hai.

Trường hợp 2: Ellipsoid được biểu diễn trên Hình 1 là ellipsoid quy chiếu chung (GRS80, WGS84, PZ-90)

Trong trường hợp này, thế trọng trường chuẩn $U_{0}$ trên mặt ellipsoid quy chiếu chung không bằng thế trọng trường thực $\overline{W_{0}}$ trên mặt geoid toàn cầu. Ngoài ra, phương trình tích phân biên theo cách tiếp cận của Molodenxkii M.X. có dạng sau:

$$
\left.\frac{\partial T_{P}}{\partial \rho}\right|_{P}-\frac{\partial \gamma}{\partial \rho} \cdot \frac{T_{P}}{\bar{\gamma}_{P}}=-\Delta g_{k k t d}+\frac{\partial \gamma}{\partial \rho} \cdot \frac{\left(U_{0}-\bar{W}_{0}\right)}{\bar{\gamma}_{P}} .
$$

Khi đó từ công thức (14) chúng ta có quan hệ $W_{P}=U_{N}+\left(\bar{W}_{0}-U_{0}\right)$. Như vậy, trong trường hợp sử dụng ellipsoid quy chiếu chung, độ cao chuẩn tại điểm $\mathrm{P}$ trên mặt vật lý được xác định trong trọng trường chuẩn của ellipsoid sao cho thế trọng trường thực $W_{P}$ tại điểm này bằng thế trọng trường chuẩn $U_{N}$ tại điểm $\mathrm{N}$ tương ứng với điểm $\mathrm{P}$ và nằm trên mặt telluroid cộng thêm đại lượng $\left(\overline{W_{0}}-U_{0}\right)$

Bản chất của các định nghĩa về độ cao chuẩn được trình bày trong hai trường hợp nêu trên được suy ra từ điều kiện Molodenxkii M.X. (14) đối với mặt telluroid. Tuy nhiên, các định nghĩa này trừu tượng và không có ý nghĩa thực tế. Trong bài báo tiếp theo, chúng ta sẽ nghiên cứu khái niệm về độ cao chuẩn khi sử dụng mặt quasigeoid.

Khi phát triển lý thuyết của mình, Molodenxkii M.X. đã dựa vào ellipsoid Trái đất trung bình thỏa mãn tính chất (11), nên mới có định nghĩa về độ cao chuẩn đã được trình bày trong trường hợp 1. Tuy nhiên, trong công trình (Yurkina M., 1996) Yurkina M. đã nhắc nhở rằng độ cao chuẩn hoàn toàn được xác định đối với ellipsoid quy chiếu chung với thế trọng trường chuẩn $U_{0}$ trên mặt ellipsoid quy chiếu chung không bằng thế trọng trường thực $\overline{W_{0}}$ trên mặt geoid toàn cầu. Mặc dù vậy, các nhà trắc địa châu Âu vẫn nhầm lẫn khi coi ellipsoid quy chiếu chung GRS80 là ellipsoid Trái đất trung bình và đã xác định Hệ độ cao Châu Âu năm 2000 (EVRF2000 - European Vertical Reference Frame of 2000) và nhận thế trọng trường thực của mặt geoid cục bộ sát nhất với mặt biển 
trung bình (NAP) tại trạm nghiệm triều Amsterdam (Hà Lan) $W_{0}=\left(U_{0}\right)_{G R S 80}=62636860,850 \mathrm{~m}^{2} \cdot \mathrm{s}^{-2}$. Sai lầm này đã được phê phán trong tài liệu (Rummel R., Heck B., 2001). Việc sửa sai đã được thực hiện đối với Khung quy chiếu độ cao Châu Âu EVRF2007 (Ihde J., 2007), theo đó mặt khởi tính của hệ độ cao Châu Âu là mặt geoid cục bộ sát nhất với mặt biển trung bình (NAP) tại trạm nghiệm triều Amsterdam (Hà Lan) với thế trọng trường thực $W_{0}=62636857,35 \mathrm{~m}^{2} \cdot \mathrm{s}^{-2}$ được công bố trong tài liệu (Bursa M., Kenyon S., Kouba J., et al., 2002).

Một vấn đề nữa cần được làm rõ là việc sử dụng công thức (6) để quy chiếu gia tốc lực trọng trường đo được $g_{P}$ trên mặt vật lý Trái đất và nhận được gia tốc lực trọng trường đo được $g_{N}$ trên mặt telluroid khi chấp nhận giả thiết rằng giữa điểm $\mathrm{P}$ và điểm $\mathrm{N}$ là "không khí tự do". Trong thực tế giữa điểm $\mathrm{P}$ và điểm $\mathrm{N}$ là lớp vỏ Trái đất với độ dày bằng dị thường độ cao $\zeta$. Tuy nhiên, do giá trị của bằng dị thường độ cao $\mathscr{\xi}$ không lớn, nên hoàn toàn có thể chấp nhận giả thiết trên. Trong trường hợp này chúng ta phải đảm bảo điều kiện: sai số trung phương $m_{g}$ của gia tốc lực trọng trường $g_{P}$ phải tương tự với sai số trung phương $m_{\hat{g}}$ của gia tốc lực trọng trường $\hat{g}_{P}$ được tính theo công thức (6), tức sai số trung phương $m_{d}$ của độ lệch $\mathrm{d}$ (7) nhỏ bỏ qua. Từ công thức $(5)$ chúng ta có công thức tính các sai số trung phương $m_{g}^{2}=m_{\hat{g}}^{2}+m_{d}^{2}$. Hiện nay sai số trung phương $m_{g}$ của gia tốc lực trọng trường đo $g_{P}$ được đánh giá bằng $\pm 1 \mathrm{mGal}$. Dựa trên nguyên tắc sai số nhỏ bỏ qua, để $m_{g}=m_{\hat{g}}$, sai số trung phương phải thỏa mãn điều kiện:

$$
m_{d} \leq \frac{m_{g}}{3}=0,333 \mathrm{mGal}
$$

Chúng ta sẽ kiểm tra giả thiết nêu trên dựa trên 38 điểm trọng lực cơ sở và hạng I phủ trùm lãnh thổ Việt Nam mà trên đó có xác định các gradients của gia tốc lực trọng trường $\partial g / \partial \rho$. Để tính các độ chênh $\mathrm{d}(7)$ trên 38 điểm trọng lực, chúng ta xác định các dị thường độ cao toàn cầu hình EGM2008 tương ứng với ellipsoid quốc tế WGS84. Các kết quả tính toán được trình bày trong Bảng 1 .

Bảng 1

\begin{tabular}{|c|c|c|c|c|c|}
\hline STT & Tên điểm trọng lực & $\begin{array}{c}\text { Độ lệch } \\
\text { mGal }\end{array}$ & STT & Tên điểm trọng lực & $\begin{array}{c}\text { Độ lệch } \\
\text { mGal }\end{array}$ \\
\hline 1 & Sa Pa & 0,126 & 20 & Hoàng Đồng & 0,365 \\
\hline 2 & Cao Bằng & 0,198 & 21 & Thọ Xuân & 0,139 \\
\hline 3 & Điện Biên & 0,152 & 22 & Quý Hòa & 0,464 \\
\hline 4 & Láng & 0.470 & 23 & Đồng Hới & 0,066 \\
\hline 5 & Vinh & 0,177 & 24 & Phú Bài & 0,042 \\
\hline 6 & Lao Bảo & 0,110 & 25 & Plei Kân & 0,082 \\
\hline 7 & Đà Nẵng & 0,026 & 26 & Plei Ku & 0,057 \\
\hline 8 & Buôn Ma Thuộc & 0,010 & 27 & Phù Cát & 0,015 \\
\hline 9 & Nha Trang & 0,068 & 28 & Tuy Hòa & 0,019 \\
\hline 10 & TP. Hồ Chí Minh & 0,060 & 29 & Diên Khánh & 0,193 \\
\hline 11 & Đảo Phú Quốc & 0,132 & 30 & Liên Nghĩa & 0,446 \\
\hline 12 & Thảo Nguyên & 0,682 & 31 & Phan Thiết & 0,040 \\
\hline 13 & Chiềng Mung & 0,337 & 32 & Vũng Tàu & 0,029 \\
\hline 14 & Hà Giang & 1,312 & 33 & An Lộc & 0,163 \\
\hline 15 & Yên Bái & 0,169 & 34 & Xa Mát & 0,059 \\
\hline
\end{tabular}




\begin{tabular}{|l|c|c|c|c|c|}
\hline 16 & Văn Dâu & 0,133 & 35 & Cần Thơ & 0,020 \\
\hline 17 & Đảo Bạch Long Vĩ & 0,167 & 36 & Châu Đốc & 0,021 \\
\hline 18 & Thịnh Long & 0,010 & 37 & Giá Rai & 0,008 \\
\hline 19 & Tiên Yên & 0,510 & 38 & Côn Đảo & 0,009 \\
\hline
\end{tabular}

Từ bảng trên chúng ta xác định sai số trung phương $m_{d}$ như sau:

$$
m_{d}= \pm \sqrt{\frac{\sum_{i=1}^{38} d_{i}^{2}}{38}}= \pm \sqrt{\frac{3,853258}{38}}= \pm 0,318 \mathrm{mGal} .
$$

Chúng ta nhận thấy rằng sai số trung phương $m_{d}$ thỏa mãn điều kiện (21), tức sai số trung phương $m_{g}$ của gia tốc lực trọng trường $g_{P}$ bằng sai số trung phương $m_{\hat{g}}$ của gia tốc lực trọng trường $\hat{g}_{P}$ được tính theo công thức (6). Như vậy các kết quả tính toán kiểm tra trên lãnh thổ Việt Nam hoàn toàn xác nhận giả thiết được sử dụng trong lý thuyết của Molodenxkii M.X..

\subsection{Các ứng dụng các kết quả giải bải toán biên hỗn hợp của Trắc địa vật lý theo cách tiếp cận của Molodenxkii M.X. trên thế giới}

Những người phê phán lý thuyết của Molodenxkii M.X. cho rằng do bề mặt Trái đất gồ ghề (rough surface), không trơn, nên không đảm bảo các điều kiện của Lipschitz để tính tích phân, ví dụ trong tài liệu (Vanícek P., R. Kingdon, M. Santos, 2012). Tuy nhiên, trong tài liệu (Sjoberg, L.E., 2013) đã chỉ ra rằng việc xác định độ cao geoid theo lý thuyết của Stokes $\mathrm{G}$. chứa các sai số phụ thuộc vào mật độ vật chất địa hình. Ở khu vực rừng núi với các độ cao từ $1-3 \mathrm{~km}$, các sai số hệ thống địa hình gây ra do các hiệu ứng địa hình trực tiếp và gián tiếp trong độ cao geoid tăng từ 1 $11 \mathrm{~cm}$. Tại đỉnh núi cao nhất, sai số hệ thống nêu trên có thể đạt tới $1 \mathrm{~m}$. Trong tài liệu (Sjoberg, L.E., 2013) cũng khẳng định rằng hệ độ cao chuẩn theo lý thuyết của Molodenxkii M.X. là sự lựa chọn tối ưu của các nước trong cả hiện tại lẫn tương lai.

Khi nói đến một quốc gia hoặc khu vực sử dụng lý thuyết của Molodenxkii M.X., chúng ta hiểu rằng quốc gia hoặc khu vực đó xây dựng hệ độ cao chuẩn và mô hình quasigeoid trên phạm vi lãnh thổ của mình. Hiện nay khu vực EU đã xây dựng hệ độ cao chuẩn khu vực EVRF2007 và mô hình quasigeoid khu vực EGG07 (the European Gravimetric Geoid Model EGG07). Mặc dù sử dụng thuật ngữ "Geoid", nhưng trong tài liệu (Denker H., J.P. Barriot, R. Barzaghi, D. Fairhead, R. Forsberg, J. Ihde, et al, 2007) khẳng định EGG07 là mô hình quasigeoid. Các nước sử dụng lý thuyết của Molodenxkii M.X. bao gồm Pháp, Đức, Thụy Điển, Ba Lan, Szech, Slovak, Bungaria, Hungary, Romania, các nước thuộc Liên Xô (cũ), Trung Quốc, Việt Nam, Lào, Campuchia .v.v...

Trong thực tế áp dụng lý thuyết của Molodenxkii M.X. luôn yêu cầu để mặt vật lý Trái đất được sử dụng làm mặt biên phải được làm trơn, tức lấy chỗ lồi đắp vào chỗ lõm. Các nước có địa hình tương đối bằng phẳng với độ cao địa hình trung bình chỉ ở mức một vài trăm mét, ví dụ Australia, Ba Lan, sử dụng mặt vật lý Trái đất thực được mô hình hóa bởi mô hình số độ cao độ phân giải 3" x 3" làm mặt biên để giải bài toán biên của Molodenxkii M.X.. Mô hình số độ cao độ phân giải cao 3" x 3" được sử dụng để tính toán các số hiệu chỉnh Faye vào các dị thường không khí tự do với mục đích loại bỏ sự tác động của các sóng ngắn của mặt geoid với biên độ nhỏ nhất từ 100 - 1000 $\mathrm{m}$ và làm trơn mặt vật lý Trái đất thực. Các nước có các khu vực rừng núi cao với độ cao địa hình trung bình của bề mặt địa hình lớn hơn $1000 \mathrm{~m}$ sử dụng mặt vật lý Trái đất trung bình (mặt Trái đất được làm trơn) được mô hình hóa bởi mô hình số độ cao độ phân giải trung bình 5 ' x 5' làm mặt biên để giải bài toán biên của Molodenxkii M.X.. Mô hình số độ cao độ phân giải trung bình $5^{\prime}$ x $5^{\prime}$ 
được sử dụng để tính toán các số hiệu chỉnh RTM vào các dị thường không khí Faye với mục đích loại bỏ sự tác động của các sóng trung của mặt geoid với biên độ nhỏ nhất từ $5-10 \mathrm{~km}$ và làm trơn mặt vật lý Trái đất trung bình (xem Hà Minh Hòa, 2018).

Bản thân Molodenxkii M.X. đã chỉ ra rằng lý thuyết của mình được sử dụng để xây dựng mô hình trọng trường Trái đất trong lần xấp xỉ lần thứ nhất. Trên cơ sở xác định được các phân bố mật độ vật chất trong lớp vỏ Trái đất sẽ làm chính xác tiếp theo mô hình trọng trường Trái đất. Theo phương hướng này, trong tài liệu (Heiskanen W.A., and Moritz H., 1967) đã đưa ra công thức chuyển dị thường độ cao (độ cao quasigeoid) $\zeta$ thành độ cao geoid $\mathrm{N}$ ở dạng sau:

$$
N=\zeta+\frac{\Delta g_{B}}{\bar{\gamma}} \cdot H^{g},
$$

ở đây $\Delta g_{B}$ - dị thường trọng lực Bouguer, $H^{g}$ - độ cao chính.

Do bài toán biên hỗn hợp được giải quyết một cách đơn trị dựa trên các trị đo trên mặt vật lý Trái đất theo cách tiếp cận của Molodenxkii M.X., nên hình thành phương hướng mới áp dụng lý thuyết của Molodenxkii M.X., theo đó đầu tiên tính toán các giá trị dị thường độ cao theo các giá trị dị thường trọng lực theo cách tiếp cận của Molodenxkii M.X.. Tiếp theo dựa trên công thức (22) chuyển đổi dị thường độ cao thành độ cao geoid. Trong tài liệu (Lemoine, F.G., Kenyon, S.C., Factor, J.K., Trimmer, R.G., Pavlis, N.K., et al, 1998) tổ chức NIMA (National Imagery and Mapping Agency) (đến năm 2003 tổ chức này đổi tên là NGA - National Geospatial-Intelligence Agency) của Mỹ đã đề xuất quy trình triển khai xây dựng mô hình trọng trường Trái đất theo phương hướng trên và dựa vào đó đã xây dựng các mô hình EGM96, EGM2008. Dựa vào quy trình trên, tổ chức NGS (National Geodetic Service) của Mỹ vào năm 2009 đã xây dựng mô hình geoid USGG2009 độ phân giải 1'x 1' của nước Mỹ (Roman D. R., Y.M. Wang, J. Saleh, X. Li, W. Waickman, 2009).

\section{Kết luận}

Trên cơ sở nghiên cứu việc giải quyết bài toán biên hỗn hợp của Trắc địa vật lý theo cách tiếp cận của Molodenxkii M.X., chúng ta đã làm rõ khái niệm về độ cao chuẩn và nhận thấy rằng lý thuyết Molodenxkii M.X. hoàn toàn khắc phục được hai vấn đề của Stokes G., bài toán biên được giải quyết trên mặt vật lý Trái đất được làm trơn. Do bài toán biên hỗn hợp của Molodenxkii M.X. được giải quyết dựa trên các kết quả đo đạc trên mặt vật lý Trái đất và trong không gian ngoài Trái đất, không dựa trên bất cứ giả thiết nào về sự phân bố mật độ vật chất trong lớp vỏ Trái đất, nên lý thuyết Molodenxkii M.X. được sử dụng ngày càng rộng rãi trên thế giới, đặc biệt kể từ khi xuất hiện công thức (22) để chuyển dị thường độ cao thành độ cao geoid. Ở Việt Nam, hệ độ cao quốc gia Hải Phòng 1972 là hệ độ cao chuẩn. Do đó để xây dựng hệ quy chiếu không gian quốc gia trong tương lai, chúng ta sẽ phải xây dựng mô hình quasigeoid quốc gia độ chính xác cao. $\bigcirc$

\section{Tài liệu tham khảo}

[1]. Bursa, M., Kenyon, S., Kouba, J., Radj, K., Vatrt, V., Vojtiskova, M., Simek, J., 2002. Word Height System specified by geopotential at tide gauge stations. IAG Symposia 124, 291-296. Springer

[2]. Denker H., J.P. Barriot, R. Barzaghi, D. Fairhead, R. Forsberg, J. Ihde, et al, 2007. The Development of the European Gravimetric Geoid Model EGG07, pp. 177 - 185. Chapter in book "Observing our Changing Earth", Michael G. Sideris (Ed.), International Association of Geodesy Symposia, Vol. 133. Proceeding of the 2007 IAG General Assembly, Perugia, Italy, July 2-3, 2007. Doi: 10.1007/978-3-540-85426-5_21, ISSN 0939-9585, Springer. 
[3]. Hà Minh Hòa, 2018. Cơ sở khoa học của việc xác định các giá trị dị thường RTM ở các khu vực rừng núi Việt Nam. Tạp chi Khoa học Đo đạc và Bản đồ, số 35, tháng 03/2018, trg. 1 - 12, ISSN: 0866- 7705.

[4]. Heiskanen, W.A., and Moritz, H., 1967. Physical Geodesy. W.H. Freeman, San Francisco, California, 364 p..

[5]. Hirt, C., Featherstone, W.E., Marti, U., 2010. Combining EGM2008 and SRTM/DTM 2006.0 residual terrain model data to improve quasigeoid computations in mountainous areas devoid of gravity data. Journal of Geodesy, 84: 557 - 567, DOI: 10.1007/s00190-010-0395-1.

[6]. Hofmann-Wellenhof B. and Moritz H., 2005. Physical Geodesy, 2nd edition, Springer, Wien - New York

[7]. Ihde, J., 2007. Final Report of IIG ICP 1.2 for the period 2003 - 2007. IAG Inter Commission Project, ICO 1.2 Vertical Reference Frames. 30 p.

[8]. Jekeli, C., 2000. Heights, the geopotential and vertical datums. Report No. 459. The Ohio State University, Columbus, $35 \mathrm{p}$.

[9]. Lemoine, F.G., Kenyon, S.C., Factor, J.K., Trimmer, R.G., Pavlis, N.K., et al, 1998. The Development of the Joint NASA GSFC and NIMA Geopotential Model EGM96, NASA Goddard Space Flight Center, NASA Technical Report NASA/TP-1996\8-206861 Greenbelt, Maryland, 20771 USA, July 1998).

[10]. Listing, J.B., 1873. Ueber unsere jetzige Kenntuts der Gestalt und der Erde. Nachrichten von der Georg - Augusts Universi3 : 33-98.

[11]. Matt, A., 2010. New Zealand Vertical Datum 2009. The New Zealand Surveyor, No. 300, pp. 5-16.

[12]. Молоденский, М. С., 1945. Основные вопросы геодезической гравиметрии. Труды ЦННИГАиК, No 42, 107 с.

Molodenxkii, M.X., 1945. Các vấn đề cơ bản của trọng lực trắc địa. Truđư TXNHIIGAiK, No42, 107 trg. (TiếngNga).

[13]. Молоденский, М. С., 1948. Внешнее гравитационное поле и фигура физической поверхности Земли. Изд. АН СССР: География и Геофизика. 12, 3, 193 - 211; Избранные труды М С Молоденского 2001. М.: Наука, 202 - 220.

Molodenxkii, M.X., 1948. Trọng trường ngoài và hình dạng mặt vật lý của Trái đất. NXB Viện Hàn lâm Khoa học Liên Xô: Địa lý và Địa vật lý. 12,3,193-211; Các công trình lựa chọn của Molodenxkii M.X. 2001. Matxcơva: Nauka, 202 - 220 trg. (TiếngNga).

[14]. NGA.STND.0036_1.0.0_WGS84. National Geospatial - Intelligence Agency (NGA) Standardization Document. Department of Defence, World Geodetic System 1984 - Its Definition and Relationships with Local Geodetic System, 2014 - 07-08, Version 1.0.0., 207 p.

[15]. Огородова, Л. В., 2010. Нормальное поле и определение аномального потенциала. Москва, МИИГАиК, 105 с.,ISBN 978-5-91188-025-5.

Ogorodova, L.V., 2010.Trường chuẩn và xác định thế dị thường. Matxcơva, MIIGAiK, 105 trg., ISBN 978-5-91188-025-5. 
[16]. Пеллинен, Л. П., 1978. Высшая геодезия (теоретичесрая геодезия). Москва. Недра, 264 c.

Pellinen, L.P., 1978. Trắc địa cao cấp (trắc địa lý thuyết). Matxcơva, Nedra, 264 trg. (Tiếng Nga).

[17]. Roman, D. R., Y.M. Wang, J. Saleh, X. Li, W. Waickman, 2009. USGG2009 \& GEOID09: New geoid height models for surveying/GPS. NOAA's National Geodetic Survey, ACSM - MARLS - UCLS - WFPS Conference 2009, 20 February 2009, Salt Lake City, UT.

[18]. Rummel, R., Heck, B., 2001. Some Critical Remarks on the Definition and Realization of the EVRS. In: Verffentlichung der Bayerischen Kommission fr die Internationale Erdmessung, M2001, Heft No 61, pp. 114-115.

[19]. Шимберов, Б. П., 1975. Теория Фигуры Земли. М., Недра, 432 с.

Simberov, B.P., 1975. Lý thuyết Hình dạng Trái đất. Matxcơva, Nedra, 325 trg. (Tiếng Nga).

[20]. Sjoberg, L.E., 2013. The geoid or quasigeoid - which reference surface should be preferred for a national height system ?. Journal of Geodetic Science, 3 (2), 103-109, doi: 10.2478/jors-20140013

[21]. Stokes, G.G., 1849. On the variation of gravity on the surface of the Earth. Transactions of the Cambridge Philosophical Society VII: 672 - 696.

[22]. Vanícek, P., R. Kingdon, M. Santos, 2012. Geoid versus quasigeoid: a case of physics and geometry. Contributions to Geophysics and Geodesy, Vol. 42/1, pp. $101-117$.

[23]. Yurkina, M., 1996. Gravity potential at the major vertical datum as primary geodetic constant. Studiageoph. etgeoid. 40, 9 - 13, Prague. $\bigcirc$

\section{Summary}

\section{A wide application of Molodenxkii's theory in the physical geodesy in the world}

\section{Ha Minh Hoa}

\section{Vietnam Institute of Geodesy and Cartography}

A key problem for construction of a national spatial reference system is to create a highly accurate quasigeoid model. However at present in Vietnam between geodesists there is mistake in definition about quasigeoid ang geoid. In this scientific article, on base of consideration of the main characters of Molodenxkii's theory we will clear definition about normal height, height anomaly and tendency of wide application of the this theory in the world. $\bigcirc$ 